\title{
Silencing miR-106b improves palmitic acid-induced mitochondrial dysfunction and insulin resistance in skeletal myocytes
}

\author{
YING ZHANG $^{1,2}$, YA-PING ZHAO ${ }^{3}$, YUAN-FU GAO ${ }^{1}$, ZHONG-MIN FAN $^{1}$, MENG-YUAN LIU ${ }^{1}$, \\ XIAO-YI CAI $^{1}$, ZHENG-KUN XIA ${ }^{1}$ and CHUN-LIN GAO ${ }^{1}$ \\ ${ }^{1}$ Department of Pediatrics, Jinling Hospital, Nanjing University, School of Medicine, Nanjing, Jiangsu 210002; \\ ${ }^{2}$ Medical College, Nankai University, Tianjin $300071 ;{ }^{3}$ Department of Pediatrics, \\ The 82nd Hospital of the People's Liberation Army, Huai'an, Jiangsu 223001, P.R. China
}

Received January 16, 2014; Accepted October 24, 2014

DOI: $10.3892 / \mathrm{mmr} .2014 .3124$

\begin{abstract}
MicroRNA-106b (miR-106b) is reported to correlate closely with skeletal muscle insulin resistance. In the current study the effect of miR-106b on palmitic acid (PA)-induced mitochondrial dysfunction and insulin resistance was investigated in $\mathrm{C} 2 \mathrm{C} 12$ myotubes via the silencing of miR-106b. MiR-106b expression was increased under PA treatment, while miR-106b loss of function improved insulin sensitivity by upregulating its target mitofusin-2 (Mfn2) in C2C12 myocytes. Furthermore, miR-106b loss of function partly improved mitochondrial morphological lesions and increased the levels of mitochondial DNA and intracellular adenosine triphosphate that had been impaired by PA exposure in C2C12 myocytes. MiR-106b loss of function attenuated the levels of intracellular reactive oxygen species (ROS), and upregulated the expression levels of the estrogen-related receptor (ERR)- $\alpha /$ peroxisome proliferative activated receptor $\gamma$ coactivator (PGC)-1 $\alpha / \mathrm{Mfn} 2$ axis under PA exposure. In addition, miR-106b negatively regulated skeletal muscle mitochondrial function and insulin sensitivity under PA-induced insulin resistance by targeting Mfn2, which may be associated with reduced ROS and upregulation of the ERR- $\alpha / \mathrm{PGC}-1 \alpha / \mathrm{Mfn} 2$ axis.
\end{abstract}

\section{Introduction}

Insulin resistance has a fundamental role in the development of obesity and type 2 diabetes mellitus $(1,2)$. Increased plasma levels of free fatty acids (FFA) are observed in these conditions, and fat accumulation in skeletal muscle is strongly associated

Correspondence to: Mr. Zheng-Kun Xia or Miss Chun-Lin Gao, Department of Pediatrics, Jinling Hospital, Nanjing University, School of Medicine, 305 Zhongshan East Road, Nanjing, Jiangsu 210002, P.R. China

E-mail: njxzk@126.com

E-mail: shuangmu34@163.com

Key words: microRNA-106b, myocytes, palmitic acid, mitochondrial dysfunction, insulin resistance with insulin resistance and a predisposition to develop type 2 diabetes mellitus $(3,4)$.

It has been reported that insulin responsiveness is impaired in human and animal models submitted to lipid infusion or a high-fat diet $(5,6)$ and in isolated skeletal muscle cells treated with FFA (2). Type 2 diabetic patients and their diabetes-prone first-degree relatives are characterized by high levels of intramyocellular lipids (IMCL) and muscular insulin resistance (7). On the contrary, endurance-trained athletes, who are among the most insulin-sensitive subjects, are additionally characterized by high IMCL levels $(7,8)$, indicating that high IMCL levels do not necessarily lead to insulin resistance. Schrauwen et al (7) have previously hypothesized that the increase in IMCL levels following endurance training served to match the training-induced increase in oxidative capacity and reliance on fat as a substrate during exercise, whereas the increase in IMCL under obesogenic/diabetogenic conditions was due to a surplus of fat availability (high plasma FFA levels, high fat diets) and was not matched by an improved oxidative capacity. Under the latter conditions, incomplete fat oxidation secondary to the overload of the tricarboxylic acid cycle by $\beta$-oxidation of FFA leads to the production of the intermediates of IMCL metabolism, including fatty acyl-CoA, diacylglycerol and ceramides, which accumulate and impede cellular insulin signaling (9). This indicates that mitochondrial dysfunction, due to an inability to accommodate the increased fat supply, may be an important event in the pathogenesis of FFA-induced insulin resistance.

However, the cellular mechanisms of the FFA-induced mitochondrial dysfunction and insulin resistance of skeletal muscle cells remain largely unknown. MicroRNAs (MiRs) represent a class of small, non-coding RNAs widely expressed in all multicellular organisms that regulate gene expression post-transcriptionally (10). Regulatory functions have been described for miRs in all tissues directly targeted by insulin, including brain, skeletal muscle, adipose and liver tissues $(11,12)$. Recent studies have shown that miR-106b is highly expressed in the skeletal muscle of diabetes patients (13) and of mice with insulin resistance induced by a 12-week high fat diet (14). In a previous study, miR-106b was discovered to induce mitochondrial dysfunction and insulin resistance in C2C12 myotubes by targeting mitofusin-2 (Mfn-2); silencing 
miR-106b improved the tumor necrosis factor (TNF)- $\alpha$-induced insulin resistance and mitochondrial dysfunction in skeletal muscle (15). In addition, it was determined that miR-106b expression levels were upregulated by palmitic acid (PA) in dose-dependent manner. In the current study, the role of miR-106b in the mitochondrial function and insulin sensitivity of skeletal myocytes was investigated under PA treatment by silencing miR-106b in C2C12 myotubes.

\section{Materials and methods}

Antibodies. Primary mouse antibodies to Mfn2 and glucose transporter 4 (GLUT4) were purchased from Abcam (Cambridge, MA, USA). The primary rabbit polyclonal peroxisome proliferative activated receptor $\gamma$ coactivator (PGC)-1 $\alpha$ antibody and estrogen-related receptor (ERR)- $\alpha$ antibody were obtained from Santa Cruz Biotechnology (Santa Cruz, CA, USA). Peroxidase-conjugated AffiniPure goat anti-rabbit secondary antibodies were purchased from Zhongshan Gold Bridge Biotechnology (Beijing, China).

Generation of stable cell lines with miR-106b loss of function. The generation of stable cell lines with miR-106b loss of function was performed as previously described (15). The miR-106b inhibitor (sponge) and a scrambled control sequence were synthesized and inserted into a LV2-pGLV lentiviral vector (GenePharma, Shanghai, China). The resulting recombinant LV2-pGLV-miR-106b inhibitor sponge plasmid and LV2-pGLV-control plasmid were confirmed by restriction endonuclease analysis and DNA sequencing. Human Embryonic Kidney (HEK)-293 cells (American Type Culture Collection, Manassas, VA, USA) were cotransfected with LV2-pGLV-miR-106b (or LV2-pGLV-control) and pLP1, pLP2 and pLP/VSVG packaging plasmids (Invitrogen Life Technologies, Carlsbad, CA, USA). The supernatant containing the lentiviral particles was harvested to determine the virus titer and used to infect murine $\mathrm{C} 2 \mathrm{C} 12$ myoblasts (American Type Culture Collection, Manassas, VA, USA). The infected cells were selected with puromycin (Sigma-Aldrich, St. Louis, MO, USA) to obtain a stable cell line.

Cell culture and treatment. Mouse $\mathrm{C} 2 \mathrm{C} 12$ myoblasts and HEK 293 cells were maintained at $37^{\circ} \mathrm{C}$ in Dulbecco's modified Eagle's medium (DMEM) containing $10 \%$ fetal bovine serum (Wisent, St. Bruno, Quebec, Canada). C2C12 cells were infected with the indicated lentivirus. At $72 \mathrm{~h}$ post-infection, cells were visualized under a fluorescence microscope (Olympus Corp., Tokyo, Japan) to observe the infection efficiency and then selected in DMEM containing $2.5 \mu \mathrm{g} / \mathrm{ml}$ puromycin for two weeks to obtain a stable cell line with miR-106b loss of function. When the $\mathrm{C} 2 \mathrm{C} 12$ cells reached confluence, the medium was changed to differentiation medium containing DMEM and 2\% horse serum (Gibco-BRL, Carlsbad, CA, USA), which was changed every other day. After five additional days, the differentiated $\mathrm{C} 2 \mathrm{C} 12$ cells had fused into myotubes. In our previous study, miR-106b expression level was not altered during C2C12 myoblasts differentiation, and the silencing of miR-106b did not affect $\mathrm{C} 2 \mathrm{C} 12$ myoblast differentiation (15). To induce insulin resistance, $\mathrm{C} 2 \mathrm{C} 12$ myotubes were serum starved for
$4 \mathrm{~h}$ and then transferred to DMEM containing 2\% bovine serum albumin (BSA) with or without PA (Sigma-Aldrich, St. Louis, MO, USA). No effect of FFA on cell viability was observed under $100 \mu \mathrm{M}$ or $200 \mu \mathrm{M}$ PA treatment for $24 \mathrm{~h}$ or $48 \mathrm{~h}$.

2-Deoxyglucose uptake assay. 2-Deoxy-D-[3H] glucose (CIC, Beijing, China) uptake was assayed as previously described (16). Following $4 \mathrm{~h}$ of serum starvation in DMEM, C2C12 myotubes were rinsed twice with 4-(2-hydroxyethyl)-1-piperazineethanesulfonic acid (HEPES)-buffered saline (20 mM HEPES, $140 \mathrm{mM} \mathrm{NaCl}$, $5 \mathrm{mM} \mathrm{KCl}, 2.5 \mathrm{mM} \mathrm{MgSO}_{4}, 1 \mathrm{mM} \mathrm{CaCl}_{2}, \mathrm{pH}$ 7.4) and stimulated with $100 \mathrm{nM}$ insulin (Peptide Institute, Osaka, Japan) for $30 \mathrm{~min}$ at $37^{\circ} \mathrm{C}$. Glucose uptake was determined by the addition of $10 \mu \mathrm{M} 2$-deoxyglucose containing $0.2 \mu \mathrm{Ci}$ $\left[{ }^{3} \mathrm{H}\right]$-2-deoxyglucose in HEPES-buffered saline for $10 \mathrm{~min}$. Ice-cold phosphate-buffered saline (PBS; $8 \%$ glucose) was used to terminate the uptake of $\left[{ }^{3} \mathrm{H}\right]$-2-deoxyglucose, and the cells were immediately washed three times with ice-cold PBS. Subsequently, the cells were lysed with $0.25 \mathrm{~N} \mathrm{NaOH}$ and the cell lysates were transferred to scintillation vials (Beckman Coulter, Brea, CA, USA) in order to measure the radioactivity using a liquid scintillation counter (Beckman Ls6500; Beckman Coulter).

Adenosine triphosphate (ATP) production. The ATP content of cells was measured with a luciferase-based luminescence assay kit (Biyuntian, Nantong, China). The cells were homogenized in an ice-cold ATP-releasing buffer (Biyuntian). Using an ATP standard, ATP concentrations were then determined with a VERITASTM microplate luminometer (Turner Biosystems, Sunnyvale, CA, USA) and normalized to protein concentrations.

Determination of intracellular reactive oxygen species (ROS) generation. Intracellular ROS generation was assayed as described previously (15). Cells were washed twice with DMEM and then incubated in pre-warmed dihydroethidium $(5 \mu \mathrm{mol} / \mathrm{l})$. After $30 \mathrm{~min}$, the cells were washed twice with DMEM, and fluorescence was immediately measured with a plate reader using fluorescence assisted cell sorting (FACS, excitation at $488 \mathrm{~nm}$, emission at $525 \mathrm{~nm}$; BD Biosciences, San Jose, CA, USA). For flow cytometry, the cells were trypsinized and centrifuged at $300 \mathrm{rpm}$ at $4^{\circ} \mathrm{C}$ for $5 \mathrm{~min}$, and then resuspended in KRP-HEPES buffer ( $\mathrm{pH} 7.4$, $0.5 \%$ BSA). The cells were analyzed with a FACScan flow cytometer using the CellQuest software (BD Biosciences, San Jose, CA, USA). The fluorescence intensity represented intracellular ROS levels.

Electron micrograph morphometry. The myocytes were collected following trypsinization, dissected and fixed in a solution of $2.5 \%$ glutaraldehyde, $1.25 \%$ paraformaldehyde and $0.03 \%$ picric acid in $0.1 \mathrm{M}$ sodium cacodylate buffer ( $\mathrm{pH} 7.4$ ). The cells were washed in $0.1 \mathrm{M}$ cacodylate buffer, postfixed with $1 \%$ osmium tetroxide and $1.5 \%$ potassium ferrocyanide for $1 \mathrm{~h}$, washed in water and stained with $1 \%$ aqueous uranyl acetate for $30 \mathrm{~min}$, followed by dehydration in a graded ethanol series (5 min in $75 \%$ ethanol, 5 min in $90 \%$ ethanol and 5 min 
Table I. Oligonucleotide sequences for primer sets used in quantitative polymerase chain reaction.

\begin{tabular}{ll}
\hline Gene & \multicolumn{1}{c}{ Sequence $\left(5^{\prime}-3\right.$ ') } \\
\hline Rn28s 1 & \\
Forward: & GGGCGGCCAAGCGTTCATAG \\
Reverse: & AGGCGTTCAGTCATAATCCCACAG \\
Probe: & TGGTAGCTTCGCCCCATTGGCTCCT \\
CYTB & \\
Forward: & TTTTATCTGCATCTGAGTTTAATCCTGT \\
Reverse: & CCACTTCATCTTACCATTTATTATCGC \\
Probe: & AGCAATCGTTCACCTCCTCTTCCTCCAC \\
PGC-1 $\alpha$ & \\
Forward: & TCTGAAAGGGCCAAACAGAG \\
Reverse: & GTAAATCACACGGCGCTCTT \\
Probe: & Roche probe \#34 \\
Mfn2 & \\
Forward: & CAGAGCAGAGCCAAACTGCT \\
Reverse: & AACATGTTGAGTTCGCTGTCC \\
Probe: & Roche probe \#20 \\
ERR- $\alpha$ & \\
Forward: & ACCTCTGGCAGTAGCTGGAG \\
Reverse: & AGCAGATGCGACACCAGAG \\
Probe: & Roche probe \#45 \\
GAPDH & \\
Forward: & AGCTTGTCATCAACGGGAAG \\
Probe: & TTTGATGTTAGTGGGGTCTCG \\
& Roche probe \#9 \\
\hline
\end{tabular}

Rn28s1, 28S ribosomal RNA; CYTB, cytochrome $b$; PGC-1- $\alpha$, peroxisome proliferative activated receptor, $\gamma$, coactivator $1 \alpha$; Mfn-2, mitofusin-2; ERR- $\alpha$, estrogen-related receptor- $\alpha$.

in $100 \%$ ethanol. The samples were subsequently embedded in TAAB Epon (Marivac Canada Inc, St. Laurent, Canada). Ultrathin sections (approximately $60 \mathrm{~nm}$ thick) were cut on a Reichert Ultracut-S Microtome (Leica, Vienna, Austria), placed onto copper grids and stained with uranyl acetate and lead citrate. The section slides were observed and photographed (magnification, x30,000) under a JEOL 1200EX microscope (JEOL, Tokyo, Japan).

Quantitative polymerase chain reaction ( $q P C R$ ) detection of nuclear and mitochondrial DNA (mtDNA). Relative amounts of nuclear DNA and mtDNA were determined by qPCR. Briefly, DNA was isolated using a DNA extraction kit (Baitaike, Beijing, China) and quantified by spectrophotometry at $260 \mathrm{~nm}$. Two primer sets were used for qPCR analysis. A 110-bp mtDNA fragment within the cytochrome $b$ gene (CYTB) was used for quantification of mtDNA. A 291-bp region of the nuclear gene for 28S ribosomal RNA ( $\mathrm{Rn} 28 \mathrm{~s} 1$ ) was used to normalize the results. qPCR was performed with an Applied Biosystems 7500 Sequence Detection System (Applied Biosystems Life Technologies, Foster City, CA, USA). The ratio of mtDNA to nuclear DNA reflects the concentration of mitochondria per cell. The sequences of primers and probes used (Shenggong, Shanghai, China) are shown in Table I.

Reverse transcription (RT)-qPCR. MiRs and total RNA from myotubes were extracted using a miRNeasy Mini Kit (Qiagen, Valencia, CA, USA) and TRIzol ${ }^{\circledR}$ reagent (Invitrogen Life Technologies). MiR-106b cDNA was generated with a reverse transcription primer provided with the TaqMan MicroRNA Reverse Transcription kit (Applied Biosystems Life Technologies), and first strand of cDNA was generated with random primers using a reverse transcription kit from Roche (Basel, Switzerland). Quantitative analysis was performed with an Applied Biosystems 7500 RT-PCR system using the following conditions: initial denaturation for $10 \mathrm{~min}$ at $95^{\circ} \mathrm{C}$, followed by 40 cycles of $15 \mathrm{sec}$ denaturation at $95^{\circ} \mathrm{C}, 30 \mathrm{sec}$ annealing at the optimal primer temperature $56^{\circ} \mathrm{C}$ and $36 \mathrm{sec}$ extension at $72^{\circ} \mathrm{C}$. Each sample was assayed in duplicate. In addition. negative controls (no template or selected untranscribed RNA) were run to ensure the absence of contamination. Analysis was performed using the $2^{-\Delta \Delta \mathrm{Ct}}$ method with U6 or GAPDH as the reference gene. Specific primers for each gene were designed to amplify a single product (Table I).

Immunoblotting. Cells were washed with ice-cold PBS and lysed with radioimmunoprecipitation assay lysis buffer (Beyotime Institute of Biotechnology, Nantong, China) for 20 min on ice. Plasma membrane proteins were extracted using the Eukaryotic Membrane Protein Extraction Reagent (Pierce, Rockford, IL, USA). Protein levels were quantified using the bicinchonic acid (BCA) protein assay kit (Pierce Biotechnology, Inc., Rockford, IL, USA) in accordance with the manufacturer's instructions. Following separation by $10 \%$ sodium dodecyl sulfate-polyacrylamide gel electrophoresis (SDS-PAGE), proteins were transferred to nitrocellulose membranes (EMD Millipore, Billerica, MA, USA). Membranes were blocked with 5\% BSA in tris buffered saline with Tween 20 (50 mM Tris, $\mathrm{pH} 7.5,150 \mathrm{mM} \mathrm{NaCl}, 0.05 \%$ Tween 20) and then incubated at $4^{\circ} \mathrm{C}$ overnight with one of the primary antibodies, followed by incubation in the presence of the secondary antibodies. The signals were detected using an enhanced chemiluminescence kit (Amersham, Piscataway, NJ, USA).

Statistical analysis. Data are expressed as the mean \pm standard deviation of at least three independent experiments. Comparisons were made using the analysis of variance for three parametric groups. Student's t-test was used for two parametric groups. $\mathrm{P}<0.05$ was considered to indicate a statistically significant difference.

\section{Results}

miR-106b expression is increased under PA treatment and miR-106b loss of function improves insulin sensitivity. Hyperlipidemia is hallmark of obesity and the insulin-resistant state. The present study sought to determine the expression pattern of miR-106b in cultured myotubes following PA exposure. PA was revealed to rapidly induced miR-106b expression in a dose-dependent manner (Fig. 1A). Treatment with $200 \mu \mathrm{M}$ PA did not alter glucose uptake from the basal 

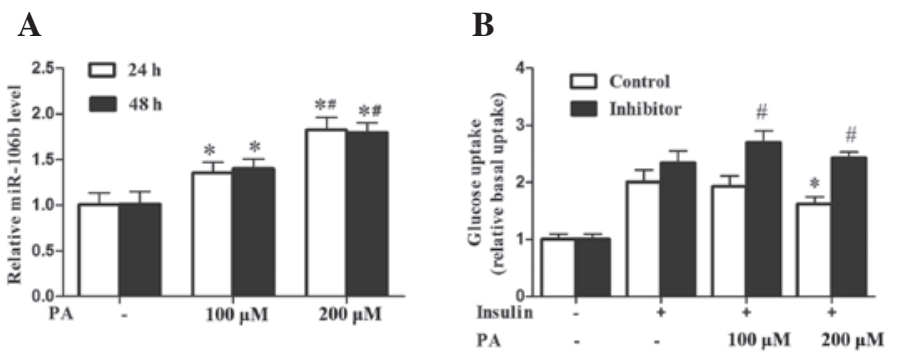

C

D

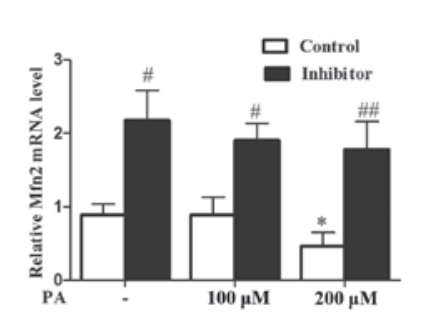

$\mathbf{E}$
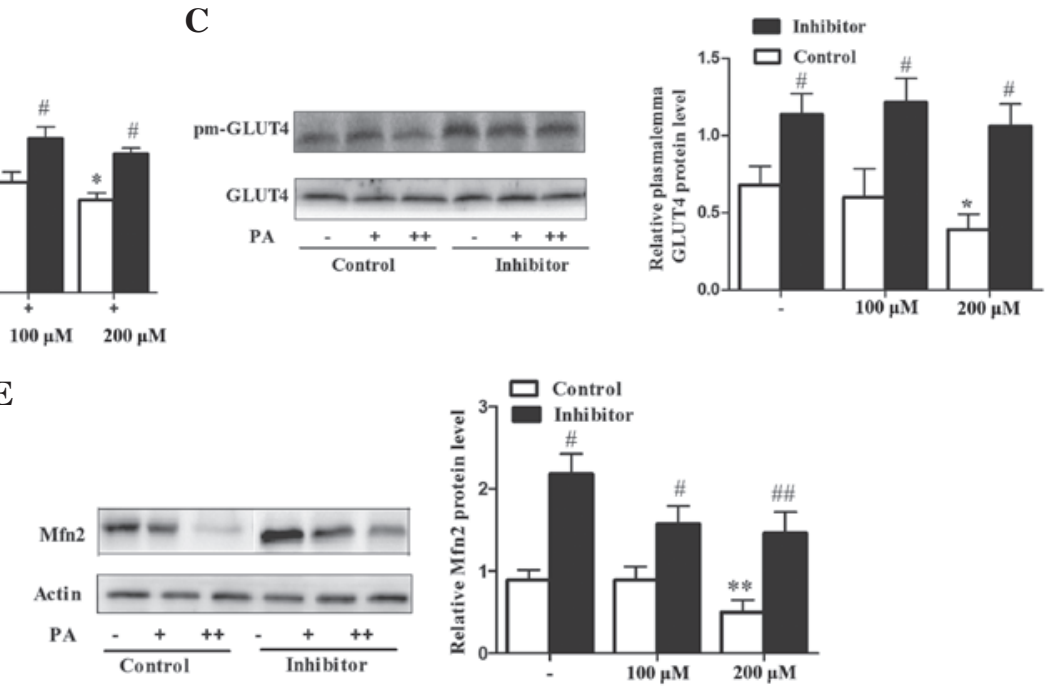

Figure 1. miR-106b expression is increased under PA treatment and negatively correlates with insulin sensitivity and Mfn2 expression. (A) PA upregulated the expression level of miR-106b in a dose-dependent manner in C2C12 myotubes. Values represent the mean \pm SD from three independent experiments ( $\mathrm{n}=3$ ). (B) Silencing of miR-106b improved insulin sensitivity under PA treatment as assessed by the 2-deoxyglucose uptake assay. Values represent the mean \pm SD from three independent experiments $(n=6)$. (C) Silencing of miR-106b increased plasma membrane GLUT4 protein level under PA treatment. Values represent the mean $\pm \mathrm{SD}$ from three independent experiments $(\mathrm{n}=3$ ). ( $\mathrm{D}$ and $\mathrm{E}$ ) Silencing of miR-106b increased Mfn2 expression under PA-induced insulin resistance (D, Mfn2 mRNA; E, Mfn2 protein). Values represent the mean \pm SD from three independent experiments $(\mathrm{n}=3)$. " $\mathrm{P}<0.05$ vs. without $\mathrm{PA}$ treatment, ${ }^{* *} \mathrm{P}<0.01$ vs. without PA treatment, ${ }^{\#} \mathrm{P}<0.05$ vs. control, ${ }^{\# \#} \mathrm{P}<0.01$ vs. control. miR, microRNA; PA, palmitic acid; SD, standard deviation; Mfn-2, mitofusin-2; GLUT4, glucose transporter type 4 .

level, however, it did significantly reduce insulin-stimulated glucose uptake by $60 \%(\mathrm{P}<0.05)$. Although no change in insulin sensitivity was observed at a concentration of $100 \mu \mathrm{M}$, PA significantly increased miR-106b expression $(\mathrm{P}<0.05)$, and this effect was more prominent at the concentration of $200 \mu \mathrm{M}$ (Fig. 1A and B). It has been confirmed that insulin-stimulated glucose uptake in skeletal muscle cells is dependent on the translocation of the insulin-responsive GLUT4 from intracellular storage compartments to the plasma membrane. PA treatment at $200 \mu \mathrm{M}$ for $24 \mathrm{~h}$ significantly reduced insulin-stimulated GLUT4 transportation to the plasma membrane in $\mathrm{C} 2 \mathrm{C} 12$ myotubes compared with that in the untreated myotubes $(\mathrm{P}<0.05)$ (Fig. 1C). Silencing miR-106b significantly increased 2-deoxyglucose uptake and GLUT4 transportation under PA treatment (Fig. 1B and C) compared with that of the controls $(\mathrm{P}<0.05)$. A previous study confirmed that miR-106b targeted Mfn2 by luciferase assay, and that miR-106b downregulated Mfn2 at the translational level in $\mathrm{C} 2 \mathrm{C} 12$ myotubes (15). In the current study, it was revealed that PA reduced the expression levels of Mfn2 mRNA and protein in $\mathrm{C} 2 \mathrm{C} 12$ myotubes, which was significantly attenuated by miR-106b loss of function (Fig. 1D and $\mathrm{E})(\mathrm{P}<0.05$ at $100 \mu \mathrm{M}$ and $\mathrm{P}<0.01$ at $200 \mu \mathrm{M})$. These results indicated that upregulation of miR-106b, which preceded a reduce in insulin sensitivity, contributed to PA-induced insulin resistance, while inhibition of miR-106b increased Mfn2 expression and improved insulin sensitivity under PA treatment.

miR-106b loss of function improves mitochondrial morphology and functions under PA-induced insulin resistance. In the above mentioned experiments, miR-106b was upregulated in parallel with the downregulation of Mfn2 by PA treatment, and the silencing of miR-106b mitigated PA-induced insulin resistance. Therefore, the current study explored whether this function was dependent on improvement of mitochondrial function. PA resulted in clear destruction of the mitochondrial ultrastructure, with the mitochondria swollen, hollow and with absent cristae (Fig. 2Ac and d). The mitochondria were condensed (smaller and higher density) and more fragmented compared with those without PA treatment. (Fig. 2Aa and b). MiR-106b loss of function partly improved mitochondrial morphological lesions impaired by PA (Fig. 2Ac and d). In addition, PA reduced production of intracellular ATP $(\mathrm{P}<0.05$ at $100 \mathrm{uM}$ and $\mathrm{P}<0.01$ at $200 \mathrm{uM})$ and mtDNA ( $<<0.05$ at $100 \mu \mathrm{M}$ and $\mathrm{P}<0.01$ at $200 \mu \mathrm{M})$ levels (Fig. 2B and C). Furthermore, miR-106b loss of function increased ATP production $(\mathrm{P}<0.05)$ and mtDNA levels $(\mathrm{P}<0.05$ at $100 \mu \mathrm{M}$ and $\mathrm{P}<0.01$ at $200 \mu \mathrm{M})$ impaired by PA treatment (Fig. 2B and C). These data suggest that silencing of miR-106b improved $\mathrm{C} 2 \mathrm{C} 12$ myotubes insulin sensitivity via protection of mitochondrial morphology and mitochondrial function.

miR-106b loss of function attenuates intracellular ROS level and improves upregulated PGC-1 $\alpha$ and ERR- $\alpha$ expression under PA-induced insulin resistance. It has been established that ROS and oxidative stress are causal factors of skeletal muscle mitochondrial dysfunction and insulin resistance $(7,17)$. The present study explored whether miR-106b protection from PA-induced mitochondrial dysfunction was associated with reduced ROS level. The results demonstrated that miR-106b silencing alleviated the ROS level $(\mathrm{P}<0.01)$, which was increased by $\mathrm{PA}$ treatment $(\mathrm{P}<0.05$ at $100 \mu \mathrm{M}$ and $\mathrm{P}<0.01$ at $200 \mu \mathrm{M})$ (Fig. 3A). PGC-1 $\alpha$ coactivated by ERR- $\alpha$, participates in the stimulation of Mfn2 expression under a variety of conditions characterized by enhanced energy expenditure $(18,19)$. Therefore, the present study analyzed whether miR-106b loss of function could affect the PGC-1 $\alpha /$ ERR- $\alpha$ axis. Treatment with $200 \mu \mathrm{M}$ PA led to a reduction in the expression levels of PGC- $1 \alpha$ mRNA and protein (Fig. 3B and D; P<0.05). PA also downregulated ERR- $\alpha$ mRNA $(\mathrm{P}<0.05$ at $100 \mu \mathrm{M}$ and $\mathrm{P}<0.01$ 
A
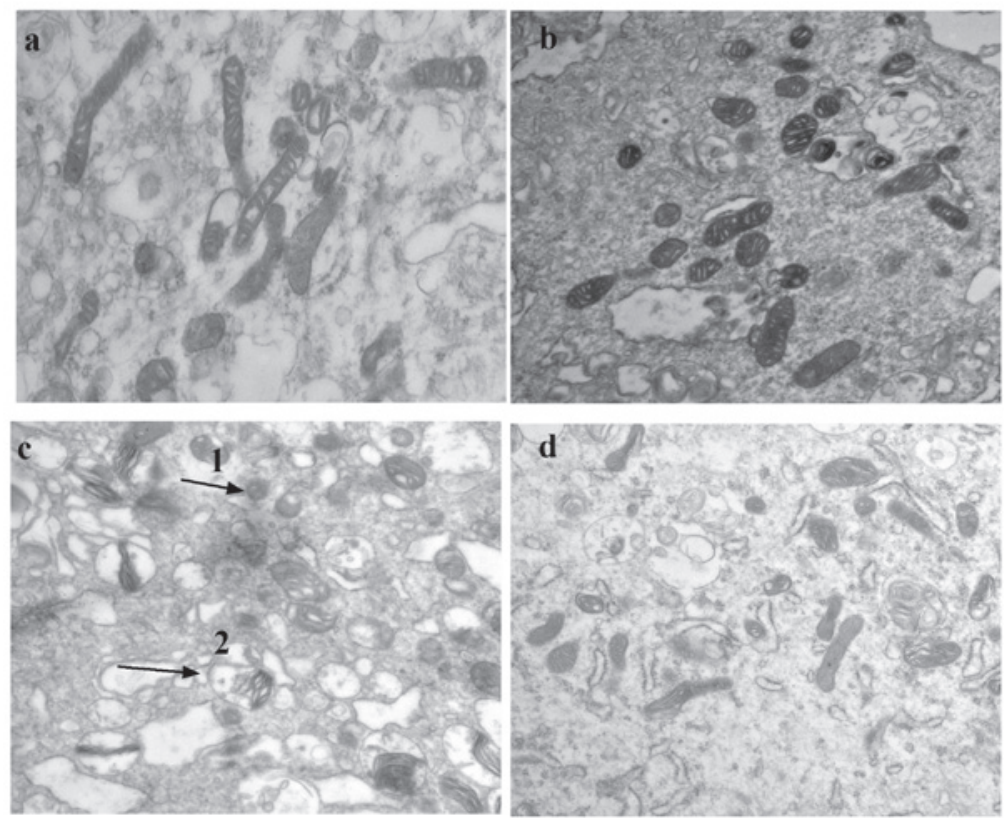

B

C

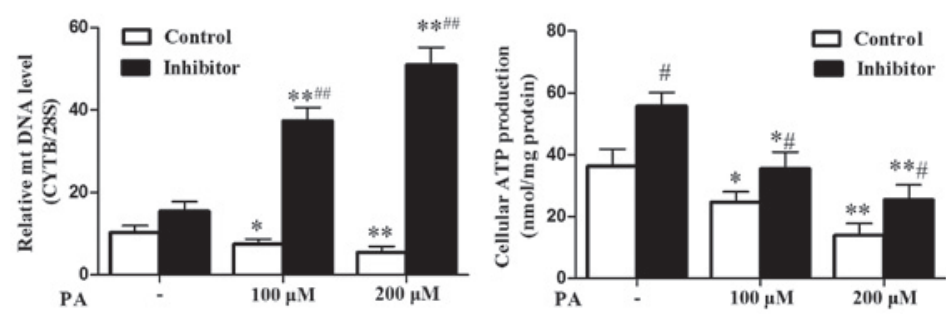

Figure 2. miR-106b loss of function improves mitochondrial morphology and mitochondrial function. (A) Changes in mitochondria morphological features of $\mathrm{C} 2 \mathrm{C} 12$ myotubes following treatment with $200 \mu \mathrm{M}$ PA for $48 \mathrm{~h}$. Ultra-structural analysis of mitochondria was performed by transmission electron microscopy (magnification, x30,000). (Aa) Control without PA treatment, (Ab) miR-106b inhibitor sponge without PA treatment, (Ac) control with 100 $\mu$ M PA treatment, (Ad) miR-106b inhibitor sponge with $100 \mu \mathrm{M}$ PA treatment). Arrow 1 indicates mitochondrial pyknosis, arrow 2 indicates mitochondrial vacuolar degeneration. (B) MiR-106b silencing increased relative mtDNA copy number, particularly under PA treatment. Values represent the mean \pm SD of three independent experiments $(n=6)$. (C) MiR-106b silencing increased cellular ATP production under PA treatment. Values represent the mean \pm SD from six independent experiments $(\mathrm{n}=6)$. ${ }^{*} \mathrm{P}<0.05$ vs. without $\mathrm{PA}$ treatment, ${ }^{* *} \mathrm{P}<0.01$ vs. without $\mathrm{PA}$ treatment, ${ }^{\#} \mathrm{P}<0.05$ vs. control. ${ }^{\# \#} \mathrm{P}<0.01$ vs. control. miR, microRNA; PA, palmitic acid; SD, standard deviation; mtDNA, mitochondrial DNA.

at $200 \mu \mathrm{M})$ and protein expression levels $(\mathrm{P}<0.05)$ (Fig. 3C and D). MiR-106b silencing upregulated PGC-1 $\alpha$ at the mRNA $(\mathrm{P}<0.01)$ and protein $(\mathrm{P}<0.05$ at $100 \mu \mathrm{M}$ and $\mathrm{P}<0.01$ at $200 \mu \mathrm{M})$ levels under PA treatment, and upregulated the expression levels of ERR- $\alpha$ mRNA $(\mathrm{P}<0.01)$ and protein $(\mathrm{P}<0.05)$ without PA treatment (Fig. 3B-D). These data suggest that inhibition of miR-106b improves mitochondrial function by reducing the levels of ROS and upregulating PGC- $1 \alpha$ and ERR- $\alpha$ expression.

\section{Discussion}

Skeletal muscle is the predominant site of glucose uptake and metabolism. It has been debated whether skeletal muscle mitochondrial dysfunction is a consequence or the cause of muscular fat accumulation. Schrauwen et al (7) reviewed these studies, which have used high-fat diets to manipulate the IMCL content and assess the impact on mitochondrial function, and came to the conclusion that rodents have the capacity to (initially) adapt mitochondrial function to a high fat intake, and that mitochondrial dysfunction only occurs as high fat diets are continued for a longer period. It was suggested that mitochondrial dysfunction may be a consequence of muscular fat accumulation, and that it develops when the protective mechanisms can no longer cope with high levels of muscular fatty acids, leading to lipotoxicity (7). It should be noted that lipid-induced mitochondrial dysfunction may lead to progressive deterioration of muscular oxidative capacity and the accumulation of lipid intermediates in skeletal muscle tissue.

It has been established that lipotoxicity, the deleterious effects of accumulating fatty acids in skeletal muscle cells, may lie at the basis of mitochondrial dysfunction; in addition to producing energy, mitochondria are the major source of reactive oxygen species (17). Fatty acids accumulating in the vicinity of the mitochondria are vulnerable to ROS-induced lipid peroxidation. Notably, increased lipid peroxidation has been reported in insulin-resistant skeletal muscle, and the mitochondrial uncoupling protein-3, which has been suggested to prevent lipid-induced mitochondrial damage by lowering ROS production, is reduced in subjects with an impaired glucose tolerance and in type 2 diabetic patients $(20,21)$. Subsequently, these lipid peroxides may have lipotoxic effects on mtDNA, RNA and the proteins of the mitochondrial machinery, leading 
A

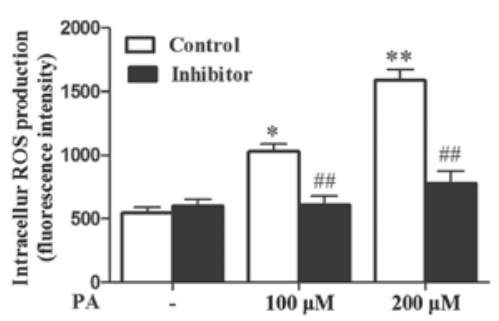

C

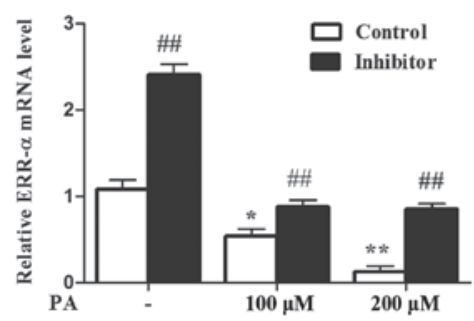

D

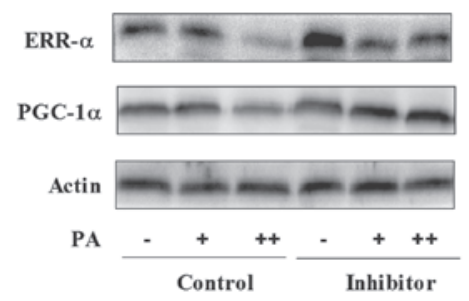

B
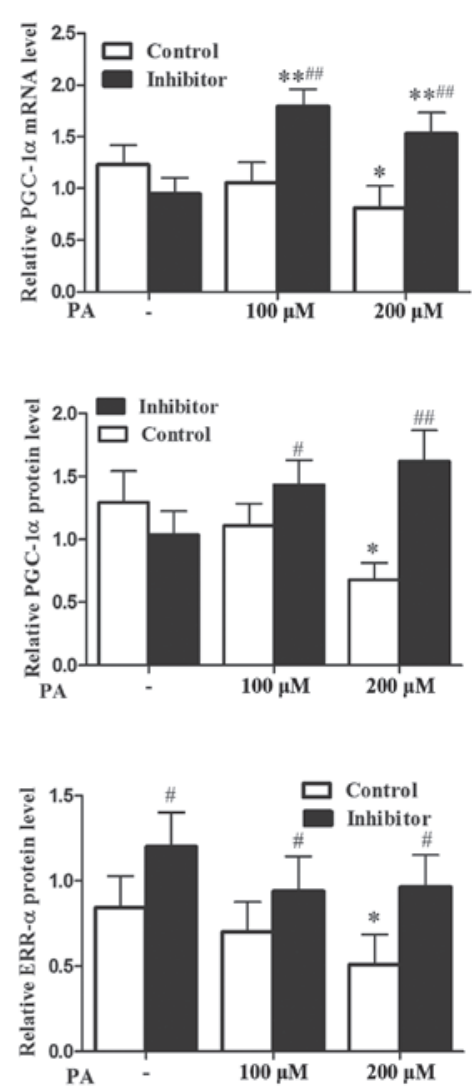

Figure 3. miR-106b loss of function attenuates intracellular ROS levels and improves upregulated PGC-1 $\alpha$ and ERR- $\alpha$ expression under PA-induced insulin resistance. (A) miR-106b loss of function attenuates intracellular ROS levels under PA treatment. Values represent the mean \pm SD from three independent experiments $(n=6)$. (B and C) PGC-1 $\alpha /$ ERR- $\alpha$ mRNA expression. Values represent the mean \pm SD from three independent experiments ( $=3$ ). (D) PGC-1 $\alpha /$ ERR- $\alpha$ protein expression. Values represent the mean \pm SD from three independent experiments $(n=3){ }^{*} \mathrm{P}<0.05$ vs. control, ${ }^{*} \mathrm{P}<0.05$ vs. without $\mathrm{PA}$ treatment, ${ }^{\# \#} \mathrm{P}<0.01$ vs. control, ${ }^{* *} \mathrm{P}<0.01$ vs. without PA treatment. miR, microRNA; ROS, reactive oxygen species; SD, standard deviation; PA, palmitic acid; PGC-1 $\alpha$, peroxisome proliferative activated receptor, $\gamma$, coactivator $1 \alpha$; ERR- $\alpha$, estrogen-related receptor- $\alpha$.

to mitochondrial dysfunction (7). Mitochondrial dynamics are associated with mitochondrial dysfunction and insulin resistance in skeletal muscle. It has been reported that PA shifts the balance toward fission in muscle cells by upregulating mitochondrion-associated dynamin-related protein 1 (Drp1) and fission 1 (Fis1), and inducing mitochondrial dysfunction and insulin resistance (22). However, there may be another mechanism of FFA-induced sketetal muscle mitochondrial dysfunction and insulin resisitance.

In a previous study, miR-106b was discovered to induce mitochondrial dysfunction and insulin resistance in $\mathrm{C} 2 \mathrm{C} 12$ myotubes by targeting mitofusin-2 (15). Mfn2 protein is a dynamin-related protein with GTPase activity anchored in the external mitochondrial membrane, and it mediates mitochondrial fusion (23). Mfn2 is abundantly expressed in skeletal muscle tissue, and it participates in the maintenance of the mitochondrial morphology and regulates the mitochondrial metabolism (19). Previous studies have shown that individuals with obesity and type 2 diabetes have reduced Mfn2 expression levels that positively correlate with insulin sensitivity $(24,25)$, and that Mfn2 knockdown in muscle cells impairs insulin signaling and results in mitochondrial dysfunction (26). Hence, we hypothesize that this protein has an important role in the etiology of mitochondrial dysfunction and insulin resistance in skeletal muscle.
In the current study, it was revealed that miR-106b expression levels were increased by PA treatment, and it was demonstrated that PA exposure blocked the insulin-stimulated glucose uptake and GLUT4 transportation of myocytes, effects that were reduced by miR-106b loss of function. Furthermore, the PA-treated cells exhibited reduced levels of ATP production and mtDNA, which suggests that they have reduced mitochondrial oxidative activity and biogenesis. These findings were in concord with those of Gao et al (27). It has been reported that Mfn2 ablation reduced mitochondrial membrane potential (26) and mtDNA levels (28), and enhanced the production of hydrogen peroxide (26) in myocytes. Similarly, the current study demonstrated that accompanied by Mfn2 upregulation, miR-106b loss of function altered mtDNA and ROS abandance in an adverse pattern under PA treatment. It has been shown that the ATP levels in Mfn2-silenced muscle cells are not altered (26), and overexpression of Mfn-2 in healthy muscle does not influence mitochondrial bioenergetics in mature mammalian skeletal muscle (29). However, the present study showed that miR-106b loss of function attenuated the reduced intracellular ATP levels in myotubes treated by PA. Concomitantly with mitochondrial dysfunction, PA induced high levels of intracellular ROS, and downregulated Mfn2 and ERR- $\alpha /$ PGC- $1 \alpha$ expression at the mRNA and protein levels. Jheng et al (22) revealed that excess PA induced mitochondrial 
fragmentation by increasing mitochondrion-associated Drp1 and Fis1, without alteration of Mfn2 and PGC-1 $\alpha$ in differentiated $\mathrm{C} 2 \mathrm{C} 12$ muscle cells. In addition, fragmented, discontinuous mitochondria were observed in $\mathrm{C} 2 \mathrm{C} 12$ myotubes treated with PA, resulting in a low fusion-to-fission ratio. This discrepancy in Mfn2 and PGC-1 $\alpha$ expression in C2C12 treated with PA may result from the treatment time, in the former study PA treatment was administered for $24 \mathrm{~h}$, but in the present study treatment was administered for $48 \mathrm{~h}$. The results of the current study demonstrated that miR-106b loss of function improves mitochondrial morphology and function, accompanied by the upregulation of Mfn2.

ERR- $\alpha$ transcription factor is a key regulator of Mfn2 transcription that recruits $\mathrm{PGC}-1 \beta$ and $\mathrm{PGC}-1 \alpha$, which are potent, positive regulators of $\mathrm{Mfn} 2$ expression in muscle cells $(18,19)$. The skeletal muscle of type 2 diabetic patients shows reduced expression of PGC-1 $\alpha$, and Mfn2, and under conditions of enhanced energy expenditure, PGC-1 $\alpha$ is induced to stimulate mitochondrial biogenesis, Mfn2 expression and mitochondrial function $(18,19,30)$. These regulatory pathways are defined by PGC- $1 \alpha /$ ERR- $\alpha / \mathrm{Mfn} 2$ and permit the activation of mitochondrial metabolism in response to energy needs. PA induced skeletal muscle insulin resistance and downregulated the expression levels of Mfn2/ERR- $\alpha /$ PGC- $1 \alpha$ at the mRNA and protein levels. The PGC-1 $\alpha /$ ERR- $\alpha / \mathrm{Mfn} 2$ signaling pathways participated in regulation of skeletal muscle mitochondrial function and insulin sensitivity. It is likely that other regulatory pathways, in addition to those mentioned, link PGC-1 $\alpha$ and Mfn2. The results revealed that $\mathrm{C} 2 \mathrm{C} 12$ myotubes transduced with the miR-106b sponge lentivirus and exposed to PA underwent a greater induction of PGC-1 $\alpha /$ ERR- $\alpha$ expression accompanied by enhanced Mfn2 expression. The same pattern of PGC-1 $\alpha /$ ERR- $\alpha$ and Mfn2 was observed in C2C12 myotubes transduced with the miR-106b sponge lentivirus and exposed to TNF- $\alpha$ (15). Coincidentally, heterozygous Mfn 2 knock-out mice previously subjected to cold for $48 \mathrm{~h}$ showed a 2 -fold greater induction of PGC-1 $\alpha$ expression in skeletal muscle compared to wild-type mice, and this increase was accompanied by enhanced Mfn2 expression (18). These results suggest that an Mfn2-induced homeostatic process regulates PGC-1 $\alpha /$ ERR- $\alpha$.

In conclusion, this study found that miR-106b loss of function improved mitochondrial function and insulin resistance, accompanied by an increase in the Mfn2 expression level following PA treatment, which may be associated with reduced ROS and upregulation of the ERR- $\alpha / \mathrm{PGC}-1 \alpha / \mathrm{Mfn} 2$ axis. These results suggest that miR-106b may represent a potential therapeutic target for the treatment of insulin resistance and type 2 diabetes. However, whether the effect of miR-106b under PA induced skeletal muscle insulin resistance is Mfn2-dependent requires further study.

\section{Acknowledgements}

This study was supported by grants from the National Natural Science Foundation of China (nos. 81100592 and 81270800).

\section{References}

1. Petersen KF and Shulman GI: Etiology of insulin resistance. Am J Med 119: S10-16, 2006.
2. Hirabara SM, Silveira LR, Abdulkader F, Carvalho CR, Procopio J and Curi R: Time-dependent effects of fatty acids on skeletal muscle metabolism. J Cell Physiol 210: 7-15, 2007.

3. McGarry JD: Banting lecture 2001: dysregulation of fatty acid metabolism in the etiology of type 2 diabetes. Diabetes 51: 7-18, 2002.

4. Silveira LR, Fiamoncini J, Hirabara SM, et al: Updating the effects of fatty acids on skeletal muscle. J Cell Physiol 217: 1-12, 2008.

5. Brehm A, Krssak M, Schmid AI, Nowotny P, Waldhausl W and Roden M: Increased lipid availability impairs insulin-stimulated ATP synthesis in human skeletal muscle. Diabetes 55: 136-140, 2006.

6. Chanseaume E, Malpuech-Brugére C, Patrac V, et al: Diets high in sugar, fat, and energy induce muscle type-specific adaptations in mitochondrial functions in rats. J Nutr 136: 2194-2200, 2006.

7. Schrauwen P, Schrauwen-Hinderling V,Hoeks J and Hesselink MK: Mitochondrial dysfunction and lipotoxicity. Biochim Biophys Acta 1801: 266-271, 2010.

8. Schrauwen-Hinderling VB, Hesselink MK, Schrauwen P and Kooi ME: Intramyocellular lipid content in human skeletal muscle. Obesity (Silver Spring) 14: 357-367, 2006.

9. Koves TR, Ussher JR, Noland RC, et al: Mitochondrial overload and incomplete fatty acid oxidation contribute to skeletal muscle insulin resistance. Cell Metab 7: 45-56, 2008.

10. Guo H, Ingolia NT, Weissman JS and Bartel DP: Mammalian microRNAs predominantly act to decrease target mRNA levels. Nature 466: 835-840, 2010.

11. Ferland-McCollough D, Ozanne SE, Siddle K, Willis AE, Bushell M: The involvement of microRNAs in Type 2 diabetes. Biochemical Society Transactions 38: 1565-1570, 2010.

12. Poy MN, Spranger M and Stoffel M: microRNAs and the regulation of glucose and lipid metabolism. Diabetes Obes Metab 9 Suppl 2: 67-73, 2007.

13. Gallagher IJ, Scheele C, Keller P, et al: Integration of microRNA changes in vivo identifies novel molecular features of muscle insulin resistance in type 2 diabetes. Genome Med 2: 9, 2010.

14. Chen GQ, Lian WJ, Wang GM, Wang S, Yang YQ and Zhao ZW: Altered microRNA expression in skeletal muscle results from high-fat diet-induced insulin resistance in mice. Mol Med Report 5: 1362-1368, 2012.

15. Zhang Y, Yang L, Gao YF, et al: MicroRNA-106b induces mitochondrial dysfunction and insulin resistance in $\mathrm{C} 2 \mathrm{C} 12$ myotubes by targeting mitofusin-2. Mol Cell Endocrinol 381: 230-240, 2013.

16. Shi CM, Wang YM, Zhang CM, et al: Knockdown of NYGGF4 (PID1) rescues insulin resistance and mitochondrial dysfunction induced by FCCP in 3T3-L1 adipocytes. Mitochondrion 12: 600-606, 2012.

17. Houstis N, Rosen ED and Lander ES: Reactive oxygen species have a causal role in multiple forms of insulin resistance. Nature 440: 944-948, 2006.

18. Soriano FX, Liesa M, Bach D, Chan DC, Palacin M and Zorzano A: Evidence for a mitochondrial regulatory pathway defined by peroxisome proliferator-activated receptor-gamma coactivator-1 alpha, estrogen-related receptor-alpha, and mitofusin-2. Diabetes 55: 1783-1791, 2006.

19. Zorzano A: Regulation of mitofusin-2 expression in skeletal muscle. Appl Physiol Nutr Metab 34: 433-439, 2009.

20. Russell AP, Gastaldi G, Bobbioni-Harsch E, et al: Lipid peroxidation in skeletal muscle of obese as compared to endurance-trained humans: a case of good vs. bad lipids? FEBS Lett 551: 104-106, 2003.

21. Schrauwen P, Mensink M, Schaart G, et al: Reduced skeletal muscle uncoupling protein-3 content in prediabetic subjects and type 2 diabetic patients: restoration by rosiglitazone treatment. J Clin Endocrinol Metab 91: 1520-1525, 2006.

22. Jheng HF, Tsai PJ, Guo SM, et al: Mitochondrial fission contributes to mitochondrial dysfunction and insulin resistance in skeletal muscle. Mol Cell Biol 32: 309-319, 2012.

23. Liesa M, Palacín M and Zorzano A: Mitochondrial dynamics in mammalian health and disease. Physiol Rev 89: 799-845, 2009.

24. Bach D, Naon D, Pich S, et al: Expression of Mfn2, the Charcot-Marie-Tooth neuropathy type 2A gene, in human skeletal muscle: effects of type 2 diabetes, obesity, weight loss, and the regulatory role of tumor necrosis factor alpha and interleukin-6. Diabetes 54: 2685-2693, 2005.

25. Mingrone G, Manco M, Calvani M, Castagneto M, Naon D and Zorzano A: Could the low level of expression of the gene encoding skeletal muscle mitofusin-2 account for the metabolic inflexibility of obesity? Diabetologia 48: 2108-2114, 2005. 
26. Sebastian D, Hernandez-Alvarez MI, Segales J, et al: Mitofusin-2 (Mfn2) links mitochondrial and endoplasmic reticulum function with insulin signaling and is essential for normal glucose homeostasis. Proc Natl Acad Sci USA 109: 5523-5528, 2012.

27. Gao X, Zhao XL, Zhu YH, et al: Tetramethylpyrazine protects palmitate-induced oxidative damage and mitochondrial dysfunction in C2C12 myotubes. Life Sci 88: 803-809, 2011.

28. Vielhaber S, Debska-Vielhaber G, Peeva V, et al: Mitofusin-2 mutations affect mitochondrial function by mitochondrial DNA depletion. Acta Neuropathol 125: 245-256, 2013.
29. Lally JS, Herbst EA, Matravadia S, et al: Over-expressing mitofusin-2 in healthy mature mammalian skeletal muscle does not alter mitochondrial bioenergetics. PLoS One 8: e55660, 2013.

30. Zorzano A, Hernández-Alvarez MI, Palacin M and Mingrone G: Alterations in the mitochondrial regulatory pathways constituted by the nuclear co-factors PGC-1alpha or PGC-1beta and mitofusin 2 in skeletal muscle in type 2 diabetes. Biochim Biophys Acta 1797: 1028-1033, 2010. 